\title{
STAR-BASED METHODS FOR PLEIADES HR COMMISSIONING
}

\author{
Sébastien Fourest, Philippe Kubik, Laurent Lebègue, \\ Cécile Déchoz, Sophie Lacherade, Gwendoline Blanchet \\ CNES 18, avenue Edouard Belin, 31401 TOULOUSE CEDEX 4 France \\ Phone: 33.(0)5.61.27.39.97 Fax: 33.(0)5.61.27.31.67 E-mail: sebastien.fourest@cnes.fr
}

ISPRS and IAA : Pléiades Inflight Calibration and Performance Assessment

KEY WORDS: Image Quality, Calibration, MTF, Focus, Geometry, Radiometry, Micro-vibrations, Star

\begin{abstract}
:
PLEIADES is the highest resolution civilian earth observing system ever developed in Europe. This imagery program is conducted by the French National Space Agency, CNES. It has been operating since 2012 a first satellite PLEIADES-HR launched on 2011 December 17th, a second one should be launched by the end of the year. Each satellite is designed to provide optical $70 \mathrm{~cm}$ resolution colored images to civilian and defense users.

Thanks to the extreme agility of the satellite, new calibration methods have been tested, based on the observation of celestial bodies, and stars in particular. It has then been made possible to perform MTF measurement, re-focusing, geometrical bias and focal plane assessment, absolute calibration, ghost images localization, micro-vibrations measurement, etc...

Starting from an overview of the star acquisition process, this paper will discuss the methods and present the results obtained during the first four months of the commissioning phase.
\end{abstract}

\section{INTRODUCTION TO PLEIADES-HR SATELLITE}

The PLEIADES program is a space Earth Observation system led by France, under the leadership of the French Space Agency (CNES). It will operate in 2012 two agile satellites designed to provide optical images to civilian and defense users.

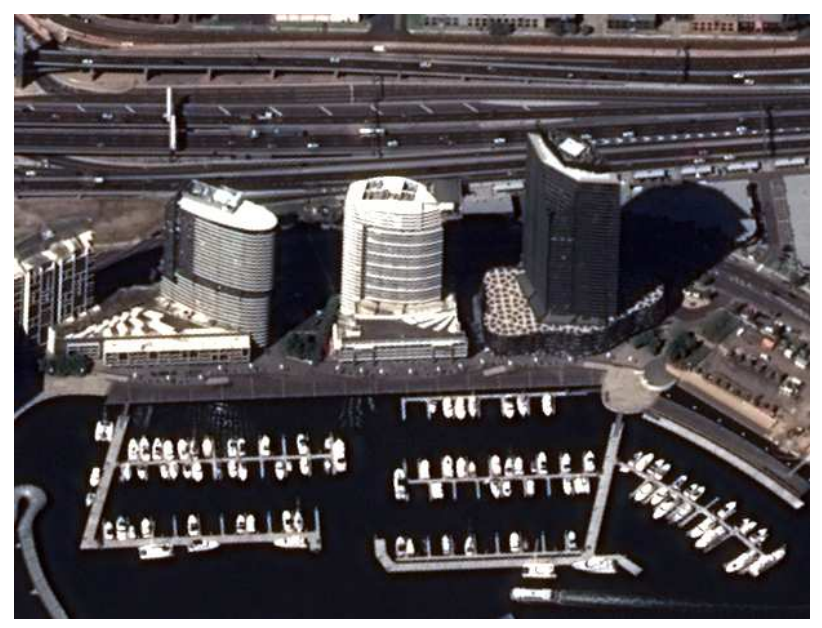

Figure 1 : Pleiades-HR image of Melbourne downtown, (C) CNES Copyright 2012

Since it was successfully launched on December 17th, 2011, Pleiades 1A high resolution optical satellite has been thoroughly tested and validated during the commissioning phase led by CNES.

Images are simultaneously acquired in Panchromatic (PA) and multi-spectral (XS) mode, which allows, in nadir acquisition condition, to deliver $20 \mathrm{~km}$ wide, false or natural colored scenes with a $70 \mathrm{~cm}$ ground sampling distance after PA+XS fusion. The scan-line is constructed by 5 detector arrays for PA and XS bands with overlapping Inter-Array-Zone (IAZ) to ensure line continuity.

Coverage will be almost world-wide with a revisit interval of $24 \mathrm{~h}$ for 2 satellites when Pleiades $1 \mathrm{~B}$ will be launched by the end of the year.

The major constraints of weight and agility led to the development of a highly compact satellite (about 1 ton weight), to minimize the moments of inertia [8]. Agility is a characteristic which allows the satellite to acquire off-nadir targets rapidly in a large flight envelope, in order to sequence numerous images. This agility is imposed by several requirements stated by the users. For instance, a 100x100 km2 zone can be acquired by the satellite from the same orbit thanks to a lateral multi-band coverage.

This agility has been largely exploited to perform numerous acquisitions of the stars to fulfill various activities during the 6 months of the commissioning phase at the Pleiades Image Calibration Center (ICC) at CNES.

The first challenge has been to find out the right stars to aim at, and the right steering, depending on the measurements to do. The Pleiades field of view being $1.6 \mathrm{deg}$ wide, the number of stars visible in one scene is small, taking into account there magnitude (Fig. 2).

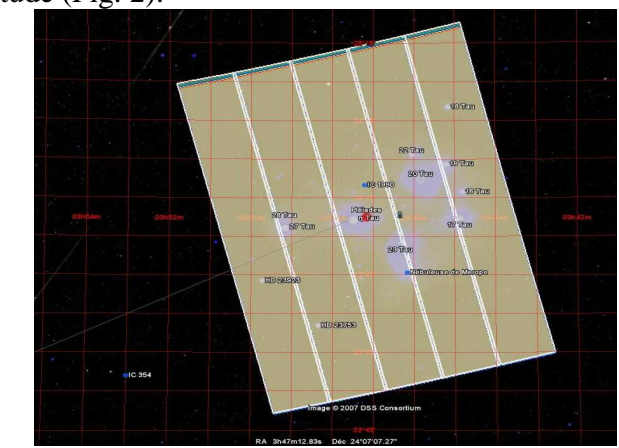

Figure 2 : example of zone of interest: the Pleiades constellation 


\section{CHOICE OF THE STARS}

\subsection{Imaging stars}

First we will focus on the range of magnitudes of the stars that can be used in our calibration techniques. Two conditions must be satisfied: detectors must not saturate, and the Signal to Noise Ratio (SNR) must be high enough in order to clearly distinguish the star.

As shown in [2], the total energy brought by a star on the detector array is given in LSB by the formula :

$E=R_{e q}($ star $) \cdot A_{k}$,

with $R_{e q}($ star $)=\left(\frac{f}{d x}\right)^{2} \cdot I_{0} \cdot 2.512^{-M a g} \cdot S p($ star $)$

where $f$ is the focal length of the instrument, $d x$ the size of a single detector, $I_{0}$ the irradiance of a star of null magnitude, Mag the magnitude of the star, $A_{k}$ the coefficient of absolute calibration [5], and $S p($ star) a coefficient taking into account the spectrum of the star (i.e. its temperature) with regard to the spectral filter of the detector. $R_{e q}$ is defined as the equivalent radiance of the star for a given spectral band of the PHR (Pleiades HR) instrument. This energy is spread over several adjacent pixels, because of the PSF of the instrument. Thus, in order to estimate the numerical count in a pixel, it has to be affected by a factor $F_{c}$, depending both of the PSF and of the position of the star with regard to the sampling grid. Indeed, if the star is imaged right in the middle of a detector, it won't produce the same numerical count than if it is imaged at the intersection of 4 pixels.

In the case of Pleiades HR, the value of this factor $F_{c}$ may vary between 0.14 and 0.34 for the panchromatic band (Fig. 3) [2].

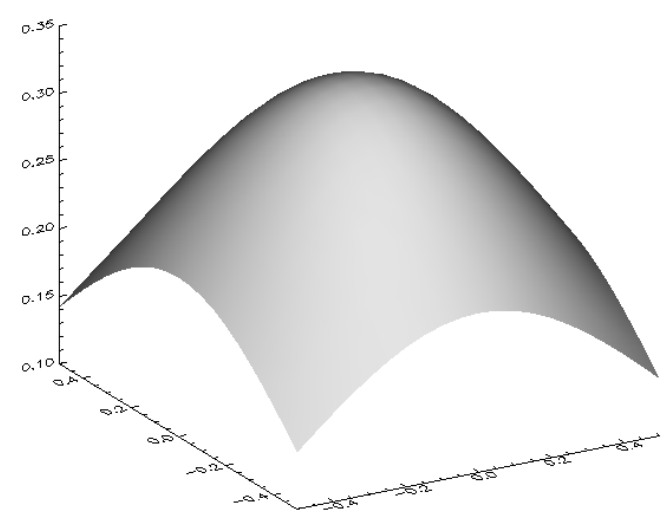

Figure $3:$ Factor Fc function of the centering of the star in the sampling grid

\subsection{Acquisition modes vs. available stars}

Depending on the type of measurement to perform, different kinds of steering can be commanded, leading to different numerical count for a same star. For a regular steering, the $A_{k}$ coefficient depends on the number of stage used in the TDI detector. In case of a "slow motion" guidance, when the scanline remains constant along the image, the stages of the TDI have to be considered as a $2 \mathrm{D}$ array, the $A_{k}$ coefficient corresponds to a single stage acquisition, and the image on the array has to be summed over all the activated stages.

In order to be compliant with radiometric constraints (no saturation and high SNR), the numerical count in each pixel must be less than 4095, and greater than 100. Taking into account the theoretical value of $A_{k}$ ( 0.85 unit per TDI stage) and the extreme values of the $F_{c}$ factor, we can assess an admissible range for the equivalent radiance $R_{e q}$, i.e. a range of magnitudes as a function of the star temperature.

For the PA band of Pleiades HR, with nominal guidance and TDI configuration (13 stages), the equivalent radiance range is from 50 to $1000 \mathrm{~W} / \mathrm{m}^{2} / \mathrm{sr} / \mu \mathrm{m}$. For a "slow motion" guidance, it goes from 450 to $9000 \mathrm{~W} / \mathrm{m}^{2} / \mathrm{sr} / \mu \mathrm{m}$.

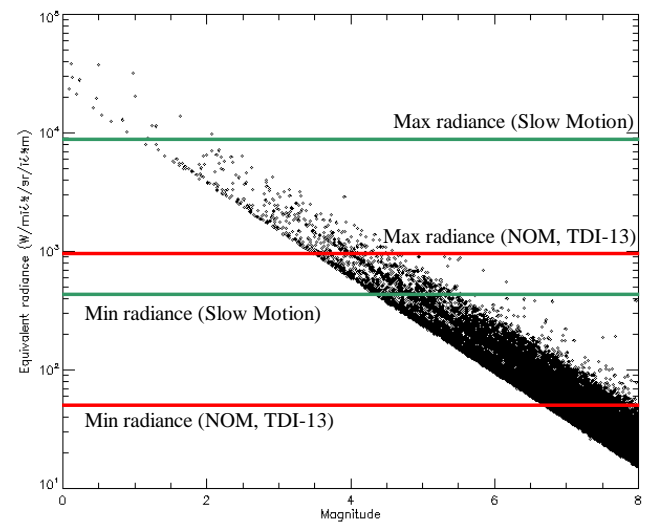

Figure 4 : equivalent radiance range (PA band) and stars magnitudes

Fig. 4 shows the equivalent radiance for the PA band as a function of the magnitude for all the stars in the Hipparcos catalog [9]. The dispersion is due to the fact that the equivalent radiance is integrated over the wavelengths of the detector's filter $(\sim 500-800 \mu \mathrm{m}$ for the panchromatic band), as the magnitude is calculated over the optical V band $(500-600 \mu \mathrm{m})$. We can see that regardless of the acquisition mode, there are a huge number of stars compliant with the radiometric constraints. Fig. 5 shows in a right ascension - declination plane the number of available stars in a single Pleiades scene $\left(1.6 * 1.6 \mathrm{deg}^{2}\right)$ for a nominal acquisition mode. The brighter the zone is, the more compliant stars we have. It's interesting to notice that the best place to aim at in the sky is the Pleiades constellation, where more than 15 stars can be correctly acquired in one scene.

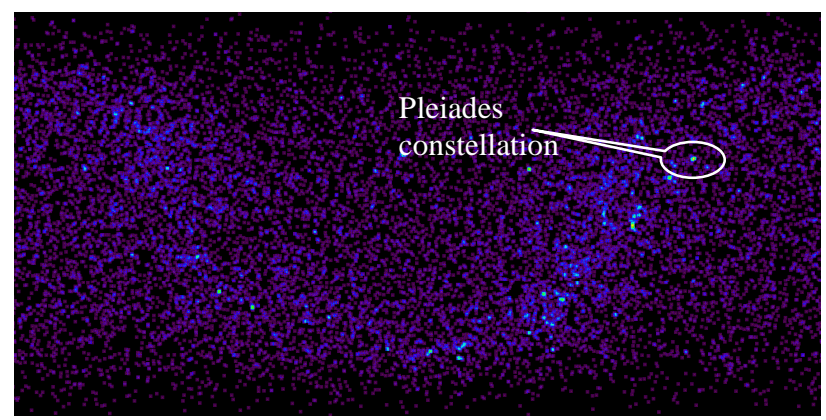

Figure 5 : compliant stars within a single PHR scene

\subsection{Simulations vs. observations}

The choice of the stars has been perfectly confirmed during the commissioning phase, the observed impulse shape being very close from the predicted one from the pre-flight theoretical and technological parameters. Fig. 6 shows the simulated and real images for two star of equivalent radiance of 51 and 812 $\mathrm{W} / \mathrm{m}^{2} / \mathrm{sr} / \mu \mathrm{m}$. The first one is shifted from the sampling grid, the second one is well centered (approximately). 


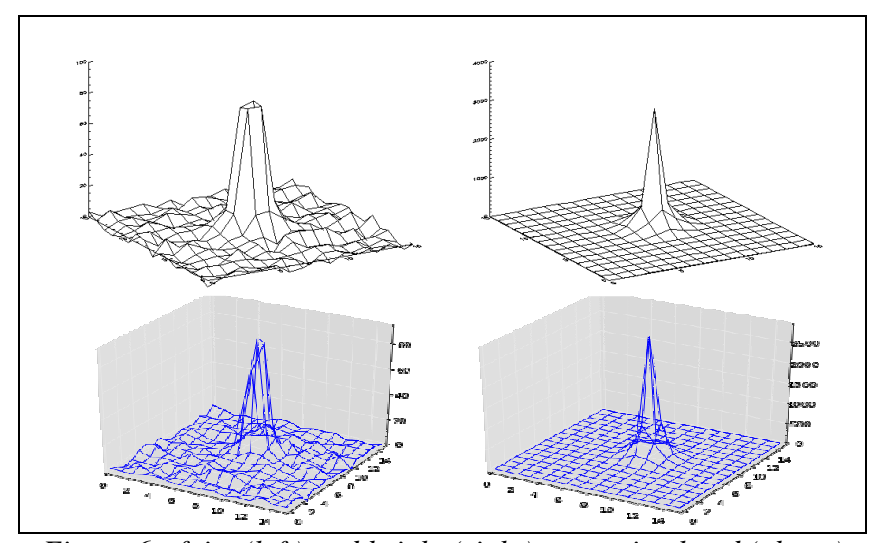

Figure $6:$ faint (left) and bright (right) stars, simulated (above) and real (below)

\section{MTF MEASUREMENT AND FOCUSING}

Since the image of a star is very close from the Point Spread Function (PSF) of the instrument, it might be natural to use the stars, as point-like sources, to assess the MTF. The main obstacle to this simple operation is the aliasing in the image, especially for the XS bands, that makes it impossible to simply do the inverse Fourier Transform of star image to obtain the MTF. Thus it is necessary to oversample the image by using numerous images and interlacing them. Thanks to a new method, based on a reasoning in the Fourier space, a high accuracy in MTF determination has been achieved.

\subsection{Methodology}

Two main steps have to be performed in order to compute precisely the MTF. The centering of the star in the sampling grid, and the interlacing of different star images. These steps are described in [2], the main guidelines are recalled here.

\subsubsection{Centering on the sampling grid}

Without any major aberration in the optical design, the imaginary part of the MTF is close to zero, thus the Fourier transform of the image of a star is real if the star is perfectly centered on the sampling grid. Otherwise, the image is shifted and the spectrum is multiplied by a phase ramp which provides imaginary values. In a case without aliasing, finding the phase ramp canceling the imaginary part of the MTF leads to the searched shift. As we always have some aliasing around Nyquist frequency it is advised to restrain the research of the phase ramp to the low frequencies, despite the fact that higher frequencies give more accurate results. A trade-off has to be made concerning the frequency limit.

The power of this method is that no hypothesis has to be made on the MTF shape as long as its imaginary part may be neglected and its real part has small values around the sampling frequency. This last condition is usually automatically fulfilled as the contribution of the detector integration to the MTF is a cardinal sine, null at this frequency.

In case the MTF is known, we can gain in accuracy by finding the shift that will simulate the best the image of the star from the theoretical PSF. This can also be done efficiently in the Fourier domain : from the oversampled MTF, we can solve the equation :

$$
F T(\text { star })=\sum_{\text {alias }} F T M * \varphi(d x, d y)
$$

where $\varphi(d x, d y)$ is the phase ramp corresponding to the unknown shift $(d x, d y)$ in the image space.

\subsubsection{Interlacing the images}

The available information is the image (or its Fourier transform) and the calculated shift from which we can determine the phase ramp. In the previous equation, we now assume that the unknown is the MTF. Aliasing is a linear operation on the MTF, and the phase ramp is known. Thus we have as many systems of linear equations as observed stars; it is possible to calculate the elements of the MTF by a mean squares method.

Each element of the Fourier transform of the image is the sum of the aliased elements of the MTF multiplied by a phase ramp. If we want to assess the MTF with an oversampling factor $s$, then $s^{2}$ terms of this MTF will contribute to each element of the FT of each image. For $N$ images of size $M x M$, we have then $N . M^{2}$ equations and $(s . M)^{2}$ unknowns, so at least $s^{2}$ images of star are needed to solve the problem. Practically, a higher number of stars is used to reduce the measurement noise.

Centering and MTF measurements can be estimated recursively in order to improve the accuracy of the results.

\subsection{MTF results}

As the programming of sky scenes have absolutely no impact on the operational activity of the satellite, many acquisitions have been performed. The usual campaigns have consisted in series of 6 to 8 scenes of the Pleiades constellation. These scenes are acquired on a single orbit, on the night side, within 3 minutes. The operational impact of these acquisitions is null. As soon as the first day of imaging with the satellite, on December $20^{\text {th }}$, a set of star images has been taken, giving a first estimation of the MTF with great accuracy. After that, around 200 image have been taken for both MTF measurements and re-focusing operations.

\subsubsection{Panchromatic band}

The raw MTF of the instrument is easy to obtain after an acquisition campaign. The brightest star (Merope) has to be removed, because it might saturate the detector. The next four ones (magnitude from 3.6 to 4.3) are kept, that is, from 24 to 32 stars per campaign. As the cut-off frequency for the PAN band is equal to the double of the Nyquist frequency, an oversampling by a factor of 2 of the MTF is enough, and 24 stars are more than enough (4 would be theoretically enough). The focus is not the same on the stars than on the ground. There is a $240 \mu \mathrm{m}$ shift between the two targets. In order to have a good estimation of the MTF on the ground, we should either focus the instrument on the stars before acquiring the images or be focused on the ground, and apply a filter to the MTF taking into account the decay due to the difference of focus. Anyway, it is necessary to have a good knowledge of the focus in order to assess the MTF on the ground from a MTF on the stars. This is why the first campaigns have given approximate values of the MTF (+/- 0.02).

Finally, the MTF measured for the panchromatic band is equal to 0.16 in both directions (Fig. 7 and Tab. 1). The accuracy is better than 0.01 .

\subsubsection{Multispectral bands}

For the XS bands, there is very little impact of the focus on the MTF. Thus, it can be directly assessed from the one computed on the stars. The main obstacle comes from the fact that this MTF is very high, as well as the cut-off frequency (around 4 times the Nyquist frequency). This implies to have many stars to oversample the PSF, with a centering accuracy that might be very approximate. To overcome this problem, a special guidance has been applied to the satellite, to slow down its pitch speed by a factor of 4 . This will naturally oversample the image 
along the columns of the image, and allow a better accuracy of the centering. Then, we can make 4 images from one by taking one row over four (Fig. 9). With this guidance, we can achieve the same MTF computation accuracy with the same number of images. It is remarkable to notice that the MTF is properly assessed for very high frequencies, up to 4 times the Nyquist frequency, and even more (Fig. 8). The accuracy is better along the columns, as this is the oversampled direction.

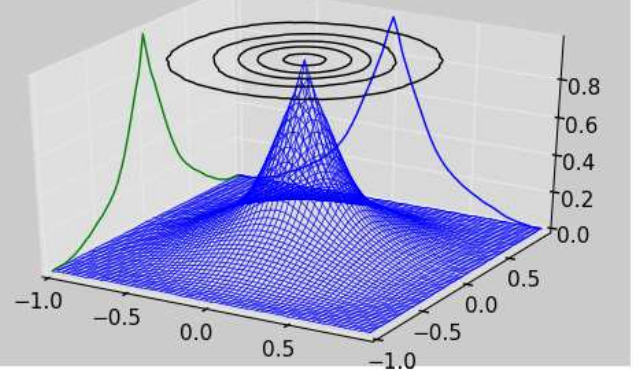

Figure $7: 2 D$ MTF for the PA band, up to $2 f_{\text {Nyquist }}$

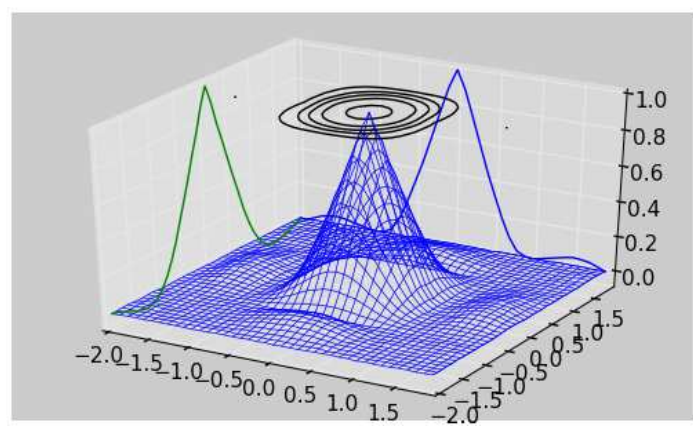

Figure $8: 2 D$ MTF for the BO band, up to $4 f_{\text {Nyquist }}$

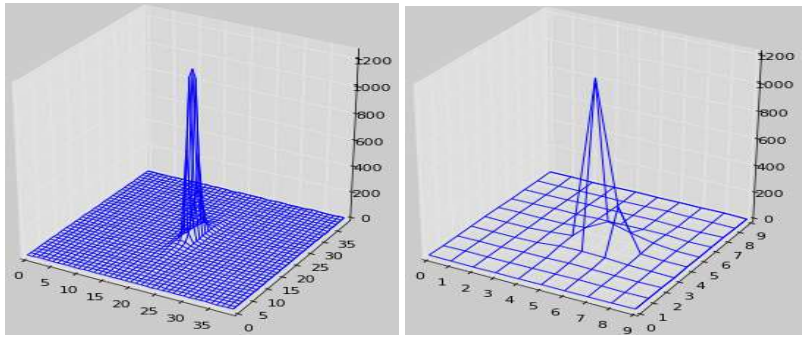

Figure 9:XS image with a slow down factor 4 (left), and one of the four extracted images (right)

This special guidance leads to a MTF where the motion blur effect doesn't appear, because the scrolling speed of the star in the focal plane is divided by 4 . It has to be simulated to obtain the real value of the MTF along the columns. Finally the MTF measured for the multispectral bands are given in Table 1.

These values (PA and XS) are compliant with the specifications, and close from the ones measured on the ground before launch.

\begin{tabular}{|l|c|c|}
\hline & $X$ & $Y$ \\
\hline PAN & 0.16 & 0.16 \\
\hline BO & 0.33 & 0.28 \\
\hline B1 & 0.33 & 0.27 \\
\hline B2 & 0.33 & 0.25 \\
\hline B3 & 0.33 & 0.26 \\
\hline
\end{tabular}

Table $1:$ MTF at Nyquist frequency

\subsection{Refocus operations}

The refocusing methodology based on the stars is detailed in [2]. To achieve this operation, a defocus campaign has to be completed : several images at different states of the focus mechanism have been taken, and the previous algorithm is run, including a model of MTF decay due to the defocus. An hypothesis has to be made about the best position for the focus mechanism, and the better this hypothesis is, the smaller the residues of the MTF computation least square are.

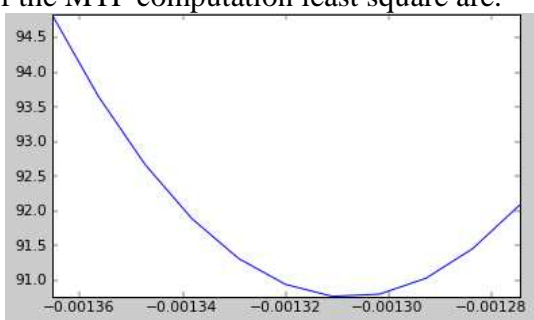

Figure 10:MTF computation residues vs. best focus hypothesis

Five campaigns have been made during the commissioning phase. For each campaign, 6 set of 5 images, taken with 6 different states of the focus mechanism, have been taken. From these campaigns, the best focus has been determined, but a shift of $240 \mu \mathrm{m}$ had to be subtracted to take into account the focus difference between stars and earth. The results consist in a control temperature of the focus ring (this is a thermal focus mechanism on Pleiades). Table 2 shows the best focus temperatures obtained after the 5 campaigns, as well as the resulting shift of the focal length in microns.

\begin{tabular}{|l|c|c|}
\hline & Temperature & Shift $(\mu \mathrm{m})$ \\
\hline $23 / 12 / 2011$ & $25^{\circ} \mathrm{C}$ & 0 \\
\hline $10 / 01 / 2012$ & $24.4^{\circ} \mathrm{C}$ & 80 \\
\hline $03 / 02 / 2012$ & $25.3^{\circ} \mathrm{C}$ & -40 \\
\hline $02 / 03 / 2012$ & $26^{\circ} \mathrm{C}$ & -140 \\
\hline $27 / 04 / 2012$ & $27^{\circ} \mathrm{C}$ & -270 \\
\hline
\end{tabular}

Table 2 : Results of the focusing campaigns

These values have been plotted over the monitoring of the focus evolution thanks to another method based on the measurement of the offset between homologous PA pixel of Inter Array Zones (IAZ, [4]). We observe a good coherence between the evolution shown by these different methods. This has confirmed that the noticed evolution of the focus since the launch is really linked to the focal length, and not to any artifact related to the monitoring method.

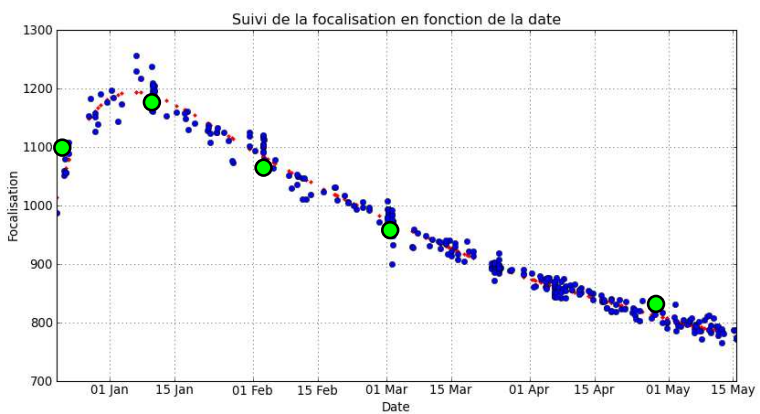

Figure 11 : defocus evolution measured in IAZ (blue) and with stars (green) 


\section{OTHER APPLICATIONS}

\subsection{Absolute calibration}

Most stars are very stable sources of light, and thanks to their magnitude and their temperature, it is possible to characterize their spectrum with rather good accuracy. By comparing the total signal integrated in the vicinity of a star in an image, and the equivalent radiance derived from the catalogue (see $\$ 2.2$ ), one can simply deduce the absolute calibration coefficient. The main challenge is that, in order to evaluate the total energy of the star in the image, we have to sum all the numerical counts of the pixels around the star, thus any bias in the darkness signal can lead to high errors in the coefficient. A primordial step is then to ensure a perfect darkness signal close to the star.

To achieve the desired accuracy, the darkness signal is computed from the image itself, as only very few pixels are impacted by the light from the stars. With a simple threshold algorithm, the darkness signal of each detector can be known. Furthermore, there is a fluctuant clock signal specific to each register (each array detector is made of 10 registers). By averaging the lines for each register, this signal can be removed. These two operations guarantee a perfect darkness in the image. As the darkness noise has a standard deviation $\sigma$, the sum of the numerical count on a small image $\mathrm{NxN}$ will have a standard deviation N. $\sigma$. Therefore, it is necessary to image several times the same star to have a good statistic on its radiometry.

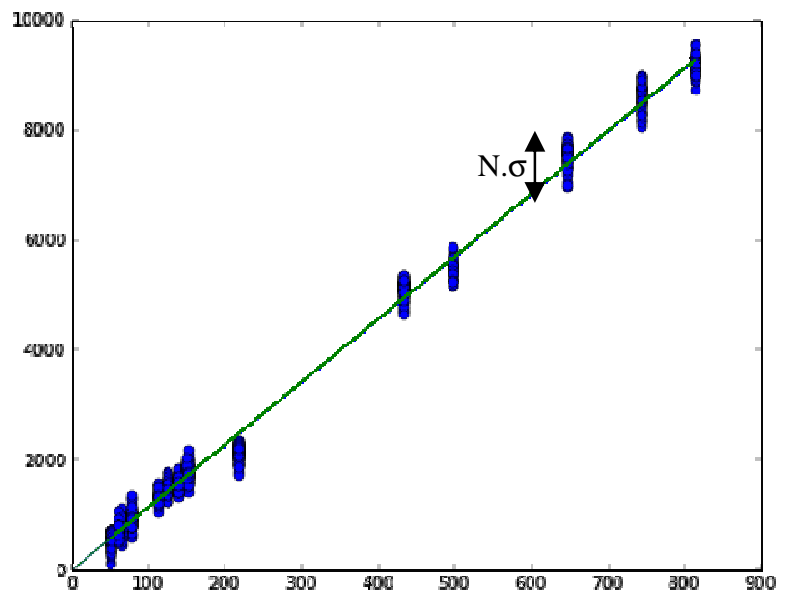

Figure 12 : Equivalent radiance vs. total numerical count

During these months, it has been noticed that the results may vary depending on the type of the star observed. It seems to be difficult to interpolate the energy of a star over the spectral range of the different bands. If it works quite well for the PA, $\mathrm{B} 0$, and B1 bands, it is less accurate for the B2 and B3 bands. Notably, the calibration has been done with hot and cold stars, and the resulting coefficient had important variations. This is why this method seems to work better for the monitoring of the absolute calibration.

Table 3 gives the absolute calibration coefficients for all spectral bands [5].

\begin{tabular}{|c|c|c|c|}
\hline & Pre-launch values & Star calibration & Other methods \\
\hline$P A$ & 11.9 & $\mathbf{1 1 . 4}$ & 11.54 \\
\hline$B 0$ & 9.1 & $\mathbf{9 . 6}$ & 9.69 \\
\hline$B 1$ & 9.63 & $\mathbf{9 . 3}$ & 9.44 \\
\hline$B 2$ & 10.74 & $\mathbf{9 . 2}$ & 10.74 \\
\hline$B 3$ & 16.66 & $\mathbf{1 2}$ & 16.38 \\
\hline
\end{tabular}

Table 3 : Absolute calibration coefficients (preliminary results)

\subsection{Micro-vibrations}

It is also possible to use star acquisitions to measure and characterize the micro-vibrations of the platform, due to the Control Moment Gyros (CMG) rotating at a constant rate.

The principle is the following : Pleiades acquires a star during few seconds in quasi-inertial pointing, with a high slow motion (slew). Thus, this star appears in the image during several rows, forming a «line» vibrating as the satellite. For each line, the position of the star in columns is computed, and this directly gives access to the profile of micro-vibration undergone by the satellite in roll axis (Fig. 13).

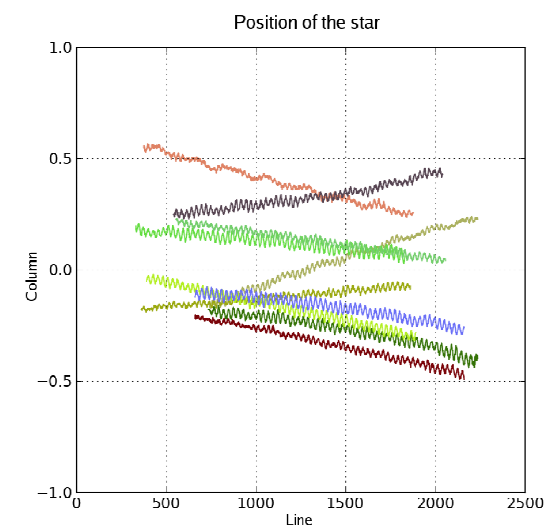

Figure 13 : position of the star measured on 10 images $(C M G$ velocity equal to $78.15 \mathrm{~Hz}$ )

To measure the position of the star in the image, the same Fourier technique is used than for the centering of the star for the MTF measurement ( $\$ 3.1 .1)$

For each spin rate of the CMGs, varying from $70.9 \mathrm{~Hz}$ to $78.4 \mathrm{~Hz}$, a dozen of acquisitions have been carried out, so as to measure the magnitude of the micro-vibrations.

It is also possible, with an excellent signal to noise ratio, to characterize the main harmonics composing the signal of microvibration and the evolution of the magnitude of these harmonics with the CMG spin rate.

It has been shown that the level of micro-vibration measured is far below the specifications and even the expected values (see [1] and [4])

\subsection{Geometric calibration}

Since the Hipparcos catalogue [9] provides stars positions with great accuracy (far better than $1 \mu \mathrm{rad}$ ), they can be seen as geometrical control points [7]. By plotting the shift between the observed star in the image and the theoretical position deduced from the attitude data and the catalogue coordinates, it is possible to evaluate the attitude biases, and as far as we have enough stars in a single scene, the attitude drifts and focal plane distortions. Fig. 14 shows for 8 images the localization error of the stars. For this purpose, even very faint stars can be used, so we can have up to 20 stars exploitable in each scene.

A 2-order distortion is obvious, as well as a yaw, a pitch drift, and a roll drift. It is also possible to detect with a good accuracy the magnification, and second and third orders in pitch and roll. Once all these defaults are removed, all the stars are colocalized with a precision better than 0.5 pixels.

This is a very promising method for the focal plane calibration. Unfortunately, the late calibration of the gyroscopes scale factors has lead to random biases in roll, pitch and yaw, that didn't permit to apply efficiently this method. 

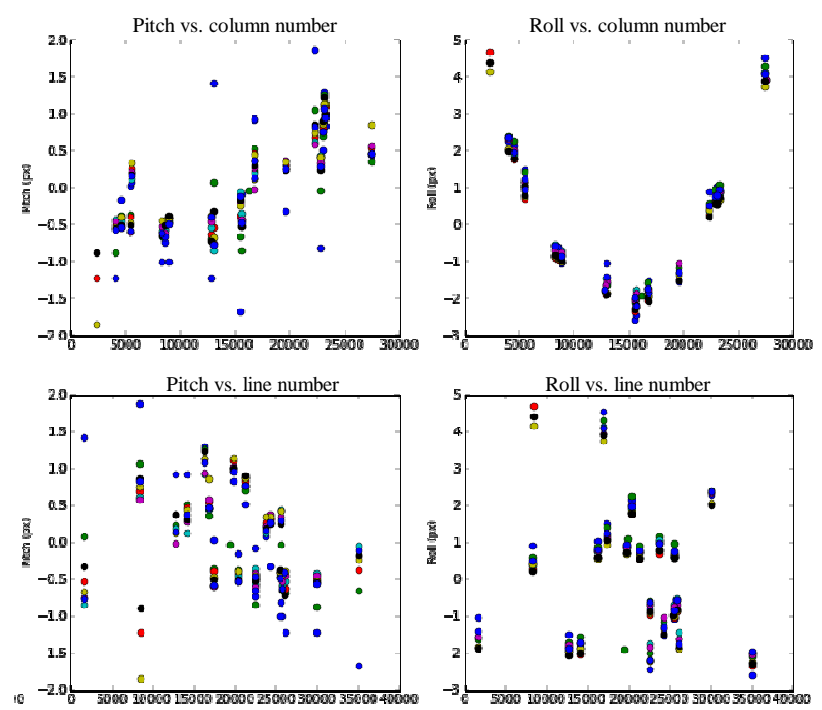

Figure 14: Geometrical effects

\subsection{Darkness currents}

So far, the darkness currents were estimated by programming images on the oceans by night [6]. A comparison has been done between this method and a computation based on star scenes, after a threshold on pixels impacted by star light. The same values have been found, with the benefit of avoiding specific programming by re-using images dedicated to MTF measurement or refocusing.

\subsection{Ghost images}

In case of very intense illumination, the light can be subjected to multiple reflections, creating ghost images. They have to be located, in order to avoid misinterpretation.

By imaging several times a very bright star, as Sirius, in different positions in the field of view, the observation of the shape of the response gives the location of this ghost. Fig. 15 presents the section of a star image at different LSB levels. On the third one, the apparition of the ghost image can be seen.
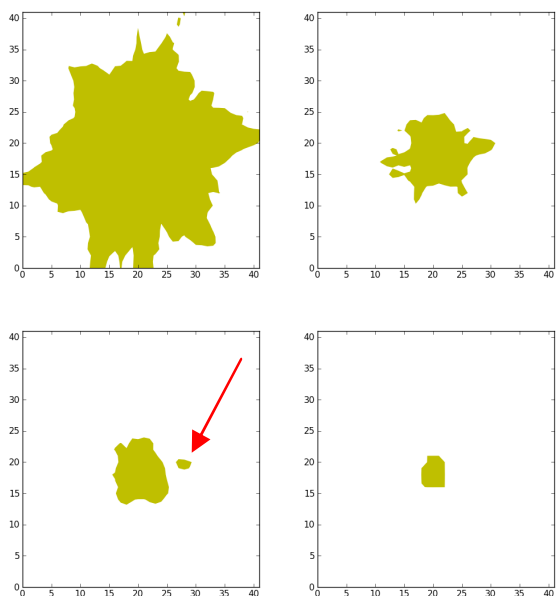

Figure 15 : Sirius sections at levels [40, 530,820,4095] LSB

\section{CONCLUSION}

On December $20^{\text {th }}$, the first image of a star taken by Pleiades has turned into reality a large number of theoretical experiments that had been dreamed for years. Since this date, many others applications have been found thanks to the ability of the satellite to aim at celestial scenes, some of them useful for all kinds of calibrations, some other just for fun and beauty : planets, 3D images of the moon and Jupiter, inter satellites shootings, ...

At all events, many time-consuming activities will now be considerably reduced thanks to the exploitation of these star images : MTF measurement and refocusing already use the stars as prime method, and absolute calibration (or at least its monitoring) and focal plane geometry could be improved after refining a few remaining points .

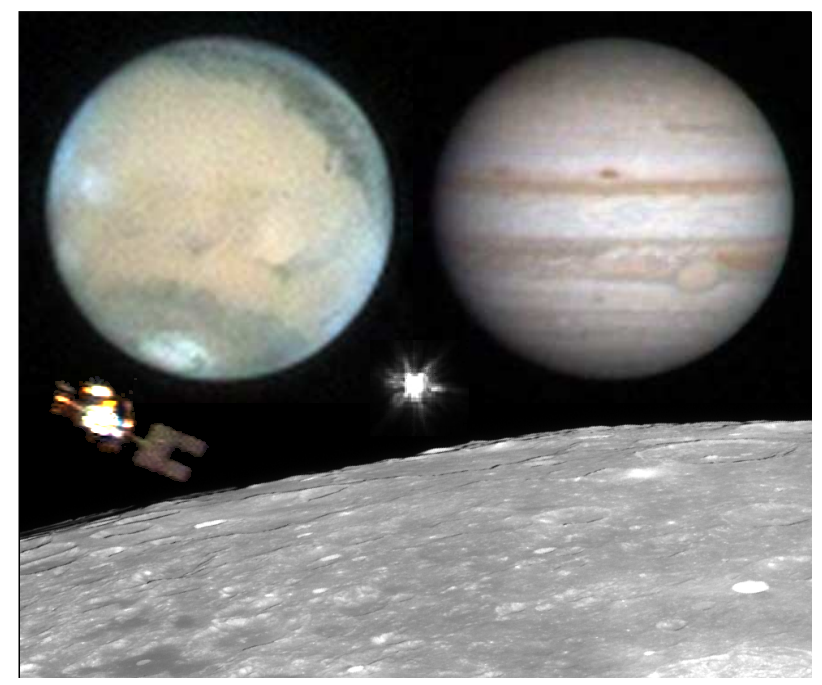

Mars, Jupiter, SPOT-5, Sirius, Moon (C) CNES Copyright 2012

\section{REFERENCES}

[1] L.Lebègue \& al, "Using Exotic Guidance for PLEIADESHR Image Quality Calibration,” ISPRS Beijing 2008.

[2] S. Fourest \& al, "Star-Based Calibration Techniques for PLEIADES-HR Satellites", CALCON Logan 2009.

[3] L.Lebègue \& al, "Pleiades-HR Image Quality commissioning foreseen methods", IGARSS Honolulu 2010.

[4] L.Lebègue \& al, "Pleiades-HR Image Quality Commissioning", ISPRS Melbourne 2012.

[5] S. Lachérade \& al, "Pleiades absolute calibration : first results", ISPRS Melbourne 2012.

[6] G. Blanchet \& al, "Pleiades-HR Innovative Techniques for Radiometric Image Quality Commissioning”, ISPRS Melbourne 2012.

[7] D. Greslou \& al, "Pleiades-HR innovative techniques for Geometric Image Quality Commissioning", ISPRS Melbourne 2012.

[8] A. Gleyzes \& al, "Pleiades system architecture and main performances", ISPRS Melbourne 2012.

[9] F. van Leeuwen, 2007 "Hipparcos, the New Reduction of the Raw Data", Astrophysics \& Space Science Library \#350.

\section{ACKNOWLEDGEMENTS}

The author would like to thank the whole team of image experts who have performed a great job, spending time, energy and enthusiasm during the 6 months of the image quality commissioning. He would also like to thank Philippe Kubik who initiated five years ago all this work, and first imagined what could be done with Pleiades and the stars. 